\title{
Thermal balance in decaying $\wedge$ cosmologies
}

\author{
D. Puy ${ }^{\star}$ \\ Geneva Observatory, University of Geneva, Chemin des Maillettes 51, 1290 Sauverny, Switzerland \\ Received 12 February 2004 / Accepted 26 February 2004

\begin{abstract}
We consider cosmological models in which the vacuum decay energy is channeled exclusively into radiation. We investigate the thermal evolution of the Universe with a variable cosmological term which varies as a function of the scale factor $a$ as $\Omega_{\Lambda}=\Omega_{\Lambda, 1}+\Omega_{\Lambda, 2} a^{-m}$. Using constraints given by the temperature measurements of the cosmic microwave background, thermal decoupling between matter and radiation is also discussed.
\end{abstract} \\ at redshifts between $z=0$ and $z=4$, we find that $\forall m \neq 0 \Omega_{\Lambda}=\Omega_{\Lambda, 0} a^{-m}$ models are clearly ruled out. The influence on the
}

Key words. cosmology: theory - cosmic microwave background

\section{Introduction}

Publications of the analysis of type Ia supernovae redshift data by the High-Z Supernova Search team (Riess et al. 1998) and the Supernova Cosmology Prospect (Perlmutter et al. 1999) have provided a strong indication that the Universe is accelerating. However, the mechanism responsible for this acceleration is still unknown.

A cosmic fluid called dark energy has been suggested by numerous authors to explain this accelerating phase. A first interpretation of this dark energy is a cosmological constant arising from vacuum energy, see Weinberg (1989), Sahni \& Starobinsky (2000). Other possibilities describing the form of dark energy were proposed such as a dynamical scalar field called quintessence (Caldwell 1998), an exotic perfect fluid called Chaplygin gas (Kamenshchik et al. 2001), a Cardassian Universe where the Friedmann Robertson Walker equation is modified (Freese \& Lewis 2002), tachyon matter (Frolov et al. 2002), four-Fermion interaction (Inagaki et al. 2003), or the Born-Infeld quantum condensate (Elizalde et al. 2003). A further approach arises from our lack of understanding of gravitational physics to explain the observed cosmic acceleration, leading to the "braneworld" theory (Dvali et al. 2000) or a modification of the Einstein-Hilbert action (Meng \& Wang 2003).

The cosmological constant has been repeatedly studied since Einstein (1917) first introduced it to obtain a static cosmological solution, see Straumann (1999). The important problem of the cosmological constant consists of explaining the observed small value, which appears to be 120 orders of magnitude smaller than one would expect based on contributions from zero-point quantum fields, see Weinberg (1989). A method of resolving this dilemna is to define a variable cosmological term. However, energy conservation would require any

\footnotetext{
^ e-mail: Denis.Puy@obs.unige.ch
}

decrease in the energy density of the vacuum to be balanced by a corresponding increase elsewhere.

In this context, Ratra \& Peebles (1998) and Peebles \& Ratra (2003) suggested that the Universe is permeated by a homogeneous scalar field, coupled weakly to matter, that rolls continuously down a potential gradient toward some minimum. Its potential energy would be converted to kinetic energy of the scalar field.

Another possibility is a decaying vacuum that convert its energy directly into radiation, see Özer \& Taha (1986), Freese et al. (1987) and Gasperini (1987). The possibility that a decaying vacuum produces matter is ruled out because the annihilation matter anti-matter (decay process does not violate baryon number) would produce a $\gamma$-ray background in excess of observed levels (see Freese et al. 1987).

The consequences of decaying $\Lambda$ cosmologies have been investigated in different contexts such as spontaneous symmetry breaking (Kazanas 1980), influence on the evolution of matter density perturbations (Silveira \& Waga 1994), on the age of the Universe (Matyjasek 1995), the statistical properties of gravitational lenses (Bloomfield-Torres \& Waga 1996), or more generally on the evolution of the scale factor with several possibilities of the functional form of $\Omega_{\Lambda}$, see Overduin \& Cooperstock (1998).

Kimura et al. (2001) then Hashimoto et al. (2003) investigated the thermal evolution of the Universe with a phenomenological decaying cosmological term. Kimura et al. (2001) found that the radiation temperature predicted from the variable $\Omega_{\Lambda}$ models is lowered significantly compared with the constant $\Omega_{\Lambda}$. Hashimoto et al. (2003) pointed out that molecular formation is shifted to earlier epochs by $\Delta a \sim 10^{-3}$ compared to the case in the standard big bang cosmology (i.e. $\Omega_{\Lambda}=$ constant). We consider the same appproach. However, prospects for constraining the cosmology by the extragalactic microwave background temperature become possible due 
to the feasibility of measuring this background temperature at redshifts between $z=0$ and $z=4$. Here we use these measurements to constrain the effects of a decaying cosmological term on the evolution of cosmic microwave radiation (CMBR) temperature.

The remainder of this paper is organized as follows: cosmological equations are presented in Sect. 2, in Sect. 3 we introduce the present cosmological parameters and our choice of parameters. In Sect. 4 we investigate the evolution of radiation temperature in which the cosmological term $\Omega_{\Lambda}$ varies. In Sect. 5 we discuss the observational constraints, then in Sect. 6 we analyse the consequences of decaying $\Lambda$ cosmologies (constrained by observations) on the thermal decoupling between matter and radiation. Our conclusions are summarized in Sect. 7.

\section{Basic dynamical properties}

Assuming the cosmological principle, it follows directly from the observed near-isotropy of the CMBR that the Universe is approximately isotropic and homogeneous. Thus with these usual assumptions, the Einstein field equations (in standard notation):

$R_{\mu \nu}-\frac{1}{2} g_{\mu \nu} R-\Lambda g_{\mu \nu}=\frac{8 \pi G}{c^{4}} T_{\mu \nu}$

associated with the Friedmann-Lemaittre-Robertson-Walker metric

$\mathrm{d} s^{2}=c^{2} \mathrm{~d} t^{2}-a(t)^{2}\left[\frac{\mathrm{d} r}{1-k r^{2}}+r^{2} \mathrm{~d}^{2} \theta+r^{2} \sin ^{2} \theta \mathrm{d}^{2} \varphi^{2}\right]$

lead to the Friedmann-Lemaître equations:

$\left(\frac{\dot{a}}{a}\right)^{2}=H^{2}=\frac{8 \pi G}{3} \rho-\frac{k c^{2}}{a^{2}}+\frac{\Lambda c^{2}}{3}$

$\frac{\ddot{a}}{a}=-\frac{4 \pi G}{3}\left(\rho+\frac{3 p}{c^{2}}\right)+\frac{\Lambda c^{2}}{2}$

where $G$ is the gravitational constant, $c$ the speed of light and $a$ the scale factor. $H$ is the Hubble parameter and $k$ the curvature constant. $\rho=\rho_{\mathrm{m}}+\rho_{\mathrm{r}}$ is the energy density associated with the pressure $p$, where $\rho_{\mathrm{m}}$ is the matter energy density and $\rho_{\mathrm{r}}$ the radiation energy density. These equations, normalised to the critical density $\rho_{\text {crit }}=3 H_{0}^{2} / 8 \pi G$, become:

$\left(\frac{\dot{a}}{a}\right)^{2}=H^{2}=H_{0}^{2}\left[\Omega_{\mathrm{r}}+\Omega_{\mathrm{m}}+\frac{\Omega_{\mathrm{K}, 0}}{a^{2}}+\Omega_{\Lambda}\right]$

$\frac{\mathrm{d} \Omega_{\mathrm{m}}}{\mathrm{d} a}+\frac{\mathrm{d} \Omega_{\mathrm{r}}}{\mathrm{d} a}+\frac{\mathrm{d} \Omega_{\Lambda}}{\mathrm{d} a}=-\frac{3}{a}\left(\frac{p / \rho_{\text {crit }}}{c^{2}}+\Omega_{\mathrm{m}}+\Omega_{\mathrm{r}}\right)$.

The curvature $\Omega_{\mathrm{K}}$ and cosmological $\Omega_{\Lambda}$ density parameter is defined by ${ }^{1}$ :

$\Omega_{\mathrm{K}}=-\frac{k c^{2}}{H_{0} a^{2}}=\frac{\Omega_{\mathrm{K}, 0}}{a^{2}}$
$\Omega_{\Lambda}=\frac{\rho_{\Lambda}}{\rho_{\text {crit }}}=\frac{\Lambda c^{2}}{3 H_{0}^{2}}$.

${ }^{1}$ The subscript 0 denotes the values at the present epoch.
The conservation of matter leads to the scale dependence:

$\Omega_{\mathrm{m}}=\frac{\rho_{\mathrm{m}}}{\rho_{\text {crit }}}=\frac{\Omega_{\mathrm{m}, 0}}{a^{3}}$.

At the present epoch all density parameters, $\Omega_{\mathrm{i}, 0}$, are correlated through the Friedmann-Lemaître condition:

$\sum_{i} \Omega_{\mathrm{i}, 0}=\Omega_{\mathrm{r}, 0}+\Omega_{\mathrm{m}, 0}+\Omega_{\mathrm{K}, 0}+\Omega_{\Lambda, 0}=1$.

From the equation of state:

- $p_{\mathrm{m}}=0$ for the non - relativistic matter and

- $p_{\mathrm{r}}=\Omega_{\mathrm{r}} \frac{\rho_{\text {crit }} c^{2}}{3}$ for radiation,

and from Eq. (6) we get the evolution of radiation density parameter:

$\frac{\mathrm{d} \Omega_{\mathrm{r}}}{\mathrm{d} a}+4 \frac{\Omega_{\mathrm{r}}}{a}=-\frac{\mathrm{d} \Omega_{\Lambda}}{\mathrm{d} a}$.

In the case $\Omega_{\Lambda}=$ constant, we deduce easily the standard evolution $\Omega_{\mathrm{r}}=\Omega_{\mathrm{r}, 0} a^{-4}$.

\section{Present cosmological parameters}

Excellent progress has been made recently toward the measurement of the Hubble constant with the Hubble Space Telescope. Combined with current estimates, the results favor the following value (see Freedman 2000):

$H_{0}=71_{-3}^{+4} \mathrm{~km} \mathrm{~s}^{-1} \mathrm{Mpc}^{-1}$.

The Far Infrared Absolute Spectrometer, (e.g. FIRAS), on board the COBE satellite (see Mather et al. 1999) has precisely measured the present radiation temperature in the frequency range $2-20 \mathrm{~cm}^{-1}$ :

$T_{\mathrm{r}, 0}=2.725 \pm 0.002 \mathrm{~K}$.

The anisotropies of the CMBR contain information about basic cosmological parameters. De Bernardis et al. (2000) with the balloon-borne observations of millimetric extragalactic radiation and geophysics (e.g. BOOMERANG), and Bennett et al. (2003) with the Wilkinson microwave anisotropy probe (e.g. WMAP satellite) found evidence for a Euclidian geometry of the Universe:

$\Omega_{\mathrm{K}, 0}=0 \pm 0.04$.

The high absolute luminosity of type Ia supernovae (used as calibrated standard candles) suggests that these objects can be seen out to large distances, making them ideal candidates for measuring and constraining cosmological parameters. The results obtained by both Riess et al. (1998) and Perlmutter et al. (1999) present direct evidence for a non-zero cosmological constant. These two experiments converge to a value for the present cosmological density parameter:

$\Omega_{\Lambda, 0}=0.73 \pm 0.04$.

(8) In our context we choose the central value at $z=0$ such as:

$H_{0}=71 \mathrm{~km} \mathrm{~s}^{-1} \mathrm{Mpc}^{-1}, T_{\mathrm{r}, 0}=2.725 \mathrm{~K}, \Omega_{\mathrm{K}, 0}=0$ and $\Omega_{\Lambda, 0}=0.73$. 


\section{Evolution of the radiation temperature in a decaying $\Lambda$ cosmology}

Numerous cosmological models with the common property of having a $\Omega_{\Lambda}$ parameter decreasing with time were proposed. Of special interest for us is the dependence of $\Omega_{\Lambda}$ on the scale factor, suggested by Gasparini (1987), Chen \& Wu (1990), Ozer (1999) and Waga (1993). Here we consider the variable- $\Lambda$ term

$\Omega_{\Lambda}=\Omega_{\Lambda, 1}+\Omega_{\Lambda, 2} a^{-m}$,

where $\Omega_{\Lambda, 1}, \Omega_{\Lambda, 2}$ and $m$ are constants. Thus the present cosmological density parameter is given by:

$\Omega_{\Lambda, 0}=\Omega_{\Lambda, 1}+\Omega_{\Lambda, 2}$.

In this context we get the evolution of the radiation density parameter (see Eq. (12)):

$\frac{\mathrm{d} \Omega_{\mathrm{r}}}{\mathrm{d} a}+4 \frac{\Omega_{\mathrm{r}}}{a}=m \Omega_{\Lambda, 2} a^{-(m+1)}$.

With the initial condition $\Omega_{\mathrm{r}}=\Omega_{\mathrm{r}, 0}$ at $a=a_{0}=1$, the superposition principle of solutions for ordinary linear differential equations easily leads to the following solution:

$\Omega_{\mathrm{r}}=\left[\Omega_{\mathrm{r}, 0}+\Omega_{\mathrm{r}, \Lambda}\right](1+z)^{4}$.

$\Omega_{\mathrm{r}, \Lambda}$ is the $\Lambda$-decay contribution to the photon background:

- $m \neq 4 \Rightarrow \Omega_{\mathrm{r}, \Lambda}=\frac{m}{4-m} \Omega_{\Lambda, 2}\left[(1+z)^{m-4}-1\right]$

- $m=4 \Rightarrow \Omega_{\mathrm{r}, \Lambda}=-4 \Omega_{\Lambda, 2} \ln (1+z)$,

where we introduce the redshift $z$

$a \equiv \frac{1}{1+z}$.

Freese et al. (1987), Overduin et al. (1993) and Matyjasek (1995) pointed out that the extra photons produced by a vacuum decay are distributed with a Planckian spectrum due to thermalization by double Compton and bremsstrahlung processes at very early times ${ }^{2}$, then keep the Planckian spectrum. Thus the radiation density parameter can be expressed by

$\Omega_{\mathrm{r}}=\frac{a_{\mathrm{r}} T_{\mathrm{r}}^{4}}{\rho_{\text {crit }} c^{2}}\left(1+f_{v}\right)=\Omega_{\mathrm{r}, 0}\left(\frac{T_{\mathrm{r}}}{T_{r, 0}}\right)^{4}$,

where $T_{\mathrm{r}}$ is the temperature of radiation, $T_{\mathrm{r}, 0}$ the present CMBR temperature and $a_{\mathrm{r}}$ the radiation density constant.

$f_{v}=\frac{7}{8} N_{v} \times\left(\frac{4}{11}\right)^{4 / 3}$

is the neutrino contribution for $N_{v}$ massless, non-degenerate neutrino types ${ }^{3}$. Finally we get the following evolution equation:

$T_{\mathrm{r}}=T_{\mathrm{r}, 0}(1+z)\left[1+\frac{\Omega_{\mathrm{r}, \Lambda}}{\Omega_{\mathrm{r}, 0}}\right]^{1 / 4}$.

\footnotetext{
${ }^{2}$ More precisely at redshift $z=6.3 \times 10^{4}\left(\Omega_{\mathrm{b}} h^{2}\right)^{-6 / 5}$, where $\Omega_{\mathrm{b}}$ is the baryonic fraction and $h=H_{0} / 100$ (see Danese \& Zotti 1977; Freese et al. 1987).

3 Mangano et al. (2002) performed a new analysis that takes into account the quantum electrodynamics correction at finite temperature to the photon and $\mathrm{e}^{ \pm}$plasma equation of state. They found $N_{v}=3.0395$, which is in agreement with the recent estimates from the primordial nucleosynthesis and WMAP results, see Barger et al. (2003).
}

Table 1. Model parameters of runs, $H_{0}$ is in $\mathrm{km} \mathrm{s}^{-1} \mathrm{Mpc}^{-1}$.

\begin{tabular}{ccccc}
\hline \hline Run & $\Omega_{\Lambda, 1}$ & $\Omega_{\Lambda, 2}$ & $m$ & $H_{0}$ \\
\hline Standard & 0.73 & 0 & 0 & 71 \\
$\mathrm{~K}$ & 0.7 & $1.8 \times 10^{-4}$ & $3 / 2$ & 60 \\
$\mathrm{~K}_{1}$ & 0.7299 & $10^{-4}$ & $3 / 2$ & 71 \\
$\mathrm{~K}_{2}$ & 0.729999 & $10^{-6}$ & 4 & 71 \\
$\mathrm{D}_{1}$ & 0.7299 & $10^{-4}$ & $-3 / 2$ & 71 \\
$\mathrm{D}_{2}$ & 0.729999 & $10^{-6}$ & $-3 / 2$ & 71 \\
\hline
\end{tabular}

Notice that in the standard case where $m=0$ (i.e. $\Omega_{\Lambda}=$ constant) we have $\Omega_{\mathrm{r}, \Lambda}=0$, and find the standard evolution $T_{\mathrm{r}, \mathrm{stan}}=(1+z) T_{\mathrm{r}, 0}$.

The analysis of the sign of $\Omega_{\Lambda, 2}$ leads to the following evolution:

- $m \Omega_{\Lambda, 2}<0 \Rightarrow \Omega_{\mathrm{r}, \Lambda}>0 \Rightarrow T_{\mathrm{r}}>T_{\mathrm{r}, \mathrm{stan}}$

- $m \Omega_{\Lambda, 2}>0 \Rightarrow \Omega_{\mathrm{r}, \Lambda}<0 \Rightarrow T_{\mathrm{r}}<T_{\mathrm{r}, \mathrm{stan}}$.

Kimura et al. (2001) analysed the thermal evolution of the Universe and showed that the radiation temperature predicted by models with a decaying cosmological term can be lowered significantly compared to the standard model (i.e. $m=0$ ). Here, we calculate the evolution of the radiation temperature with different decaying cosmological terms. In Table 1 we provide our group of parameters (the K-runs are relative to Kimura's runs).

Figure 1 compares the CMBR temperature evolution with the standard evolution. Despite that $\Omega_{\Lambda, 2}$ is very low, the difference to the standard case is clear. A larger difference is found for the $D_{1}$-model where $\Omega_{\Lambda}=0.7299+10^{-4}(1+z)^{-3 / 2}$.

\section{Observational constraints}

It is remarquable that several current observations ruled out all cosmological models in which the cosmic microwave background spectrum is non-Planckian at $z=0$. However those data are not able to constrain models with a purely blackbody spectrum but with a different $T_{\mathrm{r}}(z)$ dependence than in the standard model.

The CMBR populates excited levels of atomic and molecular species when the energy separations involved are not too different from the CMBR peak frequency. Thus the first measurement of the local CMBR temperature was made with fine structure lines in the cyanogen molecule.

Bahcall \& Wolf (1968) first suggested that this method could be extended to high redshift, where the background radiation temperature is larger, using atomic fine-structure transitions in absorbing clouds toward high-redshift quasars.

Transitions of neutral carbon and $\mathrm{C}^{+}$are particularly well suited for this. Thus it is (in theory) possible to estimate the CMBR temperature from this method. However the fine-structure fine levels can be populated by several processes, mainly collisions with hydrogen atoms and electrons, and pumping due to the local ultraviolet radiation and to the CMBR. The different contributions can be estimated once 


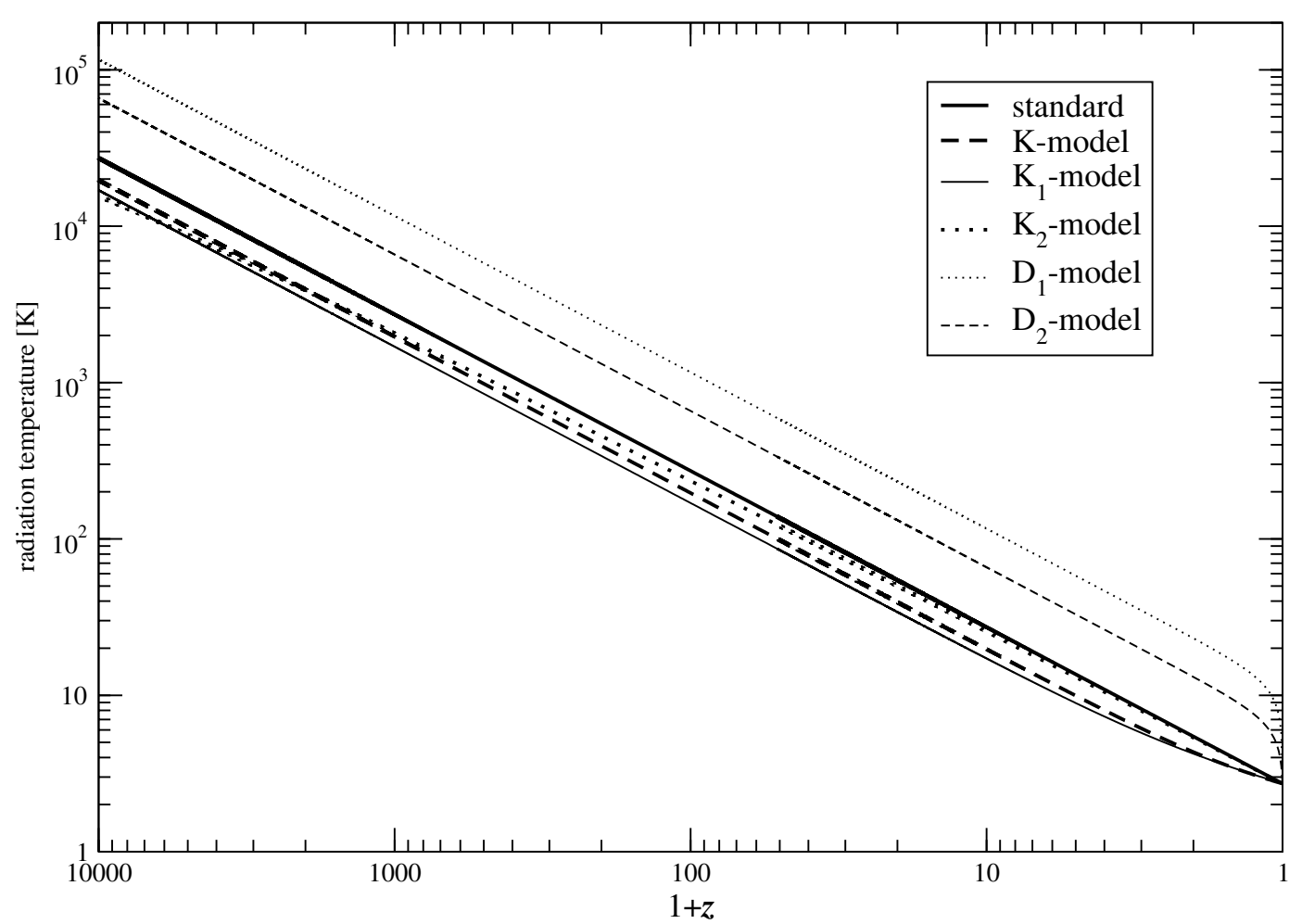

Fig. 1. Evolution of the radiation temperature for different decaying $\Lambda$-cosmologies.

the temperature and the particle density are known (see discussions in Levshakov et al. 1999 on the measurability of kinetic temperature from absorption-line spectra). Generally an upper limit on $T_{\mathrm{CMBR}}$ is obtained by assuming that the CMBR is the only excitation process at work. The measurements have been attempted several times, but have been generally limited by the resolution and signal-to-noise available in reasonable exposure times.

Songaila et al. (1994) reported the detection of absorption from the first fine-structure level of neutral carbon atoms in a cloud towards the quasar Q1331+170. The population ratio yields a temperature of

$T_{\text {Songaila }}=7.4 \pm 0.8 \mathrm{~K}$ at $z=1.776$,

assuming that no other significant sources of excitation are present. We take this range for the CMBR temperature, even though these values could be considered to be an upper limit.

Ge et al. (1997) presented detections of absorption from the ground state and excited states of $\mathrm{C}$ in the damped Ly $\alpha$ system of the quasar QSO 0013-004. From the population ratio of the excited state to the ground state, they derive an estimate for the CMBR temperature:

$T_{\mathrm{Ge}}=7.9 \pm 1.0 \mathrm{~K}$ at $z=1.9731$.

Srianand et al. (2000) detected the absorption lines from the first and second fine-structure levels of neutral carbon atoms in an isolated cloud of gas towards the quasar PKS1232+0815; they found

$6 \mathrm{~K}<T_{\text {Srianand }}<14 \mathrm{~K}$ at $z=2.3371$.

From the analysis of the $\mathrm{C}^{+}$fine-structure population ratio in the damped Ly $\alpha$ system towards the quasar Q0337-3819,
Molaro et al. (2002) derive an upper bound on the CMBR temperature

$T_{\text {Molaro }}=12.1_{-3.2}^{+1.7} \mathrm{~K}$ at $z=3.025$.

The possibility of determining the CMBR temperature from measurements of the Sunyaev-Zel'dovich (SZ) effect in the galaxy cluster has been suggested by the group of Melchiorri, see Fabbri et al. (1978). The steep frequency dependence of the change, due to the SZ effect, and the weak dependence of the ratios of intensity changes measured at two frequencies allow the estimation of the CMBR temperature at the redshift of the cluster. Recently Battistelli et al. (2003) deduced the CMBR temperature using data of the Coma cluster and Abell 2163 over four bands at radio and microwave frequencies with the millimeter and infrared Testa Grigia observatory (e.g. MITO). They found the following radiation temperature:

$T_{\text {Melchiorri2 }}=3.377_{-0.102}^{+0.101} \mathrm{~K}$ at $z=0.203$

$T_{\text {Melchiorri1 }}=2.789_{-0.065}^{+0.080} \mathrm{~K}$ at $z=0.0231$.

Thus we have six observables of the radiation temperature at different redshifts. Figure 2 compares the evolution of radiation temperature of our preceeding models with the observational value of the CMBR temperature.

Figure 2a gives the measurements of CMBR temperature at redshifts between $z=0$ and $z=4$, the lower panel gives the measurements, with the SZ effect, between the redshifts $z=0$ and $z=0.25$. We see that in some models, for example the $\mathrm{D}_{1}$-model and $\mathrm{D}_{2}$-model, radiation temperature is not included in the domain of observational estimates. Thus it is necessary to adjust the parameters to reconcile the decaying- $\Lambda$ model with the observations. 

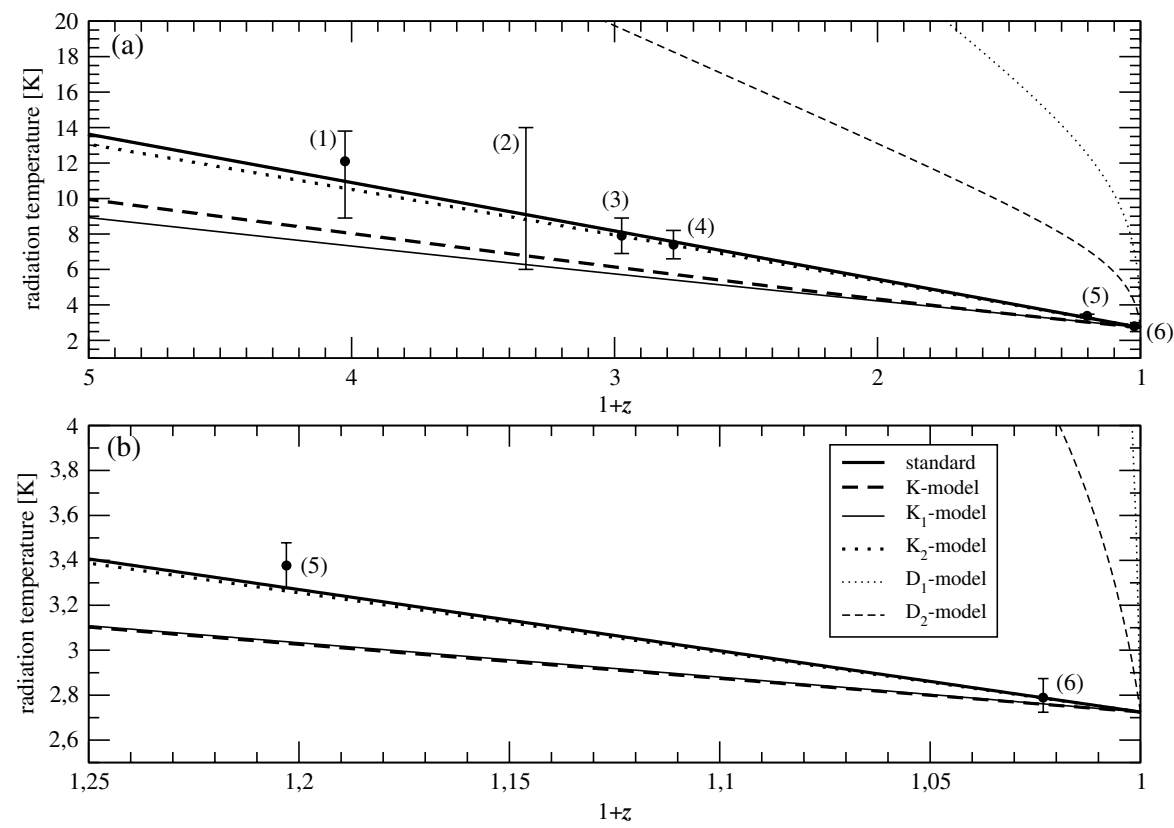

Fig. 2. Evolution of the radiation temperature for different decaying $\Lambda$-cosmologies; a) shows the evolution between $1+z=1$ and $1+z=5$, b) is relative to the range $1+z=1$ and $1+z=1.25$. Current measurements of some extragalactic cosmic microwave background temperatures are labeled as follows: (1) $T_{\text {Molaro }}$, (2) $T_{\text {Srianand }}$, (3) $T_{\mathrm{Ge}}$, (4) $T_{\text {Songaila }}$, (5) $T_{\text {Melchiorri1 }}$, (6) $T_{\text {Melchiorri2 }}$.

The six preceeding observational measurements impose a range of validity, at the observation redshift $z_{\mathrm{obs}}$, on the radiation temperature:

$T_{\mathrm{obs}}^{-}<T_{\mathrm{r}}<T_{\mathrm{obs}}^{+}$

We introduce the $\Omega_{\mathrm{obs}}^{-}$and $\Omega_{\mathrm{obs}}^{+}$quantities:

$\Omega_{\mathrm{obs}}^{-}=\Omega_{\mathrm{r}, 0}\left[\left(\frac{T_{\mathrm{obs}}^{-}}{T_{\mathrm{r}, 0}\left(1+z_{\mathrm{obs}}\right)}\right)^{4}-1\right]$

$\Omega_{\mathrm{obs}}^{+}=\Omega_{\mathrm{r}, 0}\left[\left(\frac{T_{\mathrm{obs}}^{+}}{T_{\mathrm{r}, 0}\left(1+z_{\mathrm{obs}}\right)}\right)^{4}-1\right]$.

Thus, for each observable we have $\Omega_{\mathrm{obs}}^{-}<0$ and $\Omega_{\mathrm{obs}}^{+}>0$. From Eqs. (26) and (35) we get the following condition between $\Omega_{\Lambda, 2}$ and $m$ :

$\Omega_{\Lambda, 2}^{-}<\Omega_{\Lambda, 2}<\Omega_{\Lambda, 2}^{+}$,

where the bounds $\Omega_{\Lambda, 2}^{+}$and $\Omega_{\Lambda, 2}^{-}$are such as

$\bullet m<0$ :

$\Omega_{\Lambda, 2}^{-}=\frac{4-m}{m\left[\left(1+z_{\mathrm{obs}}\right)^{m-4}-1\right]} \Omega_{\mathrm{obs}}^{-}<0$

$\Omega_{\Lambda, 2}^{+}=\frac{4-m}{m\left[\left(1+z_{\mathrm{obs}}\right)^{m-4}-1\right]} \Omega_{\mathrm{obs}}^{+}>0$;

- $m>0$ and $m \neq 4$ :

$\Omega_{\Lambda, 2}^{-}=\frac{4-m}{m\left[\left(1+z_{\mathrm{obs}}\right)^{m-4}-1\right]} \Omega_{\mathrm{obs}}^{+}<0$

$\Omega_{\Lambda, 2}^{+}=\frac{4-m}{m\left[\left(1+z_{\mathrm{obs}}\right)^{m-4}-1\right]} \Omega_{\mathrm{obs}}^{-}>0$; $\bullet \quad m=4$ :

$\Omega_{\Lambda, 2}^{-}=-\frac{\Omega_{\mathrm{obs}}^{+}}{4 \ln \left(1+z_{\mathrm{obs}}\right)}<0$
$\Omega_{\Lambda, 2}^{+}=-\frac{\Omega_{\mathrm{obs}}^{-}}{4 \ln \left(1+z_{\mathrm{obs}}\right)}>0$.

Figures 3 and 4 show the bounds on $\Omega_{\Lambda, 2}$ deduced from the six observational constraints. The left panels give $\Omega_{\Lambda, 2}^{-}$, the right panels indicate $\Omega_{\Lambda, 2}^{+}$. The shaded region marks the non-allowed parameters $\left(m, \Omega_{\Lambda, 2}\right)$.

The Melchiorri measurements with the Coma cluster at $z=0.0231$ (see Figs. $4 \mathrm{c}$ and $4 \mathrm{~d}$ ) are the most restrictive. In Fig. 5 we summarize the allowed parameters regions. We get the following constraint:

$\forall|m| \geq 1,\left|\Omega_{\Lambda, 2}\right| \geq 10^{-4}$ is not allowed.

Moreover cosmological parameters such as $\Omega_{\Lambda}=\Omega_{\Lambda, 0} a^{-m}$ with $m \neq 0$ are clearly ruled out.

\section{Thermal decoupling}

The thermal history of the early Universe depends on the tight coupling between radiation and matter resulting from Thomson scattering. The evolution of the gas temperature $T_{\mathrm{m}}$ is governed by the equation (see Kompaneets 1957; Peebles 1968):

$\frac{\mathrm{d} T_{\mathrm{m}}}{\mathrm{d} t}=-2 H T_{\mathrm{m}}+\frac{8}{3} \frac{\sigma_{\mathrm{T}} a_{\mathrm{r}}}{m_{\mathrm{e}} c} T_{\mathrm{r}}^{4}\left(T_{\mathrm{r}}-T_{\mathrm{m}}\right) \frac{n_{\mathrm{e}}}{n_{\mathrm{b}}}$,

where $\sigma_{\mathrm{T}}$ is the Thomson cross section and $m_{\mathrm{e}}$ the mass of the electron. $n_{\mathrm{e}}$ and $n_{\mathrm{b}}$ are the numerical density, i.e. number of particles per $\mathrm{cm}^{-3}$, for electrons and for baryons. We neglect the energy transfer via the molecular transitions and the enthalpies of reaction (see Puy et al. 1993; Pfenniger \& Puy 2003). 

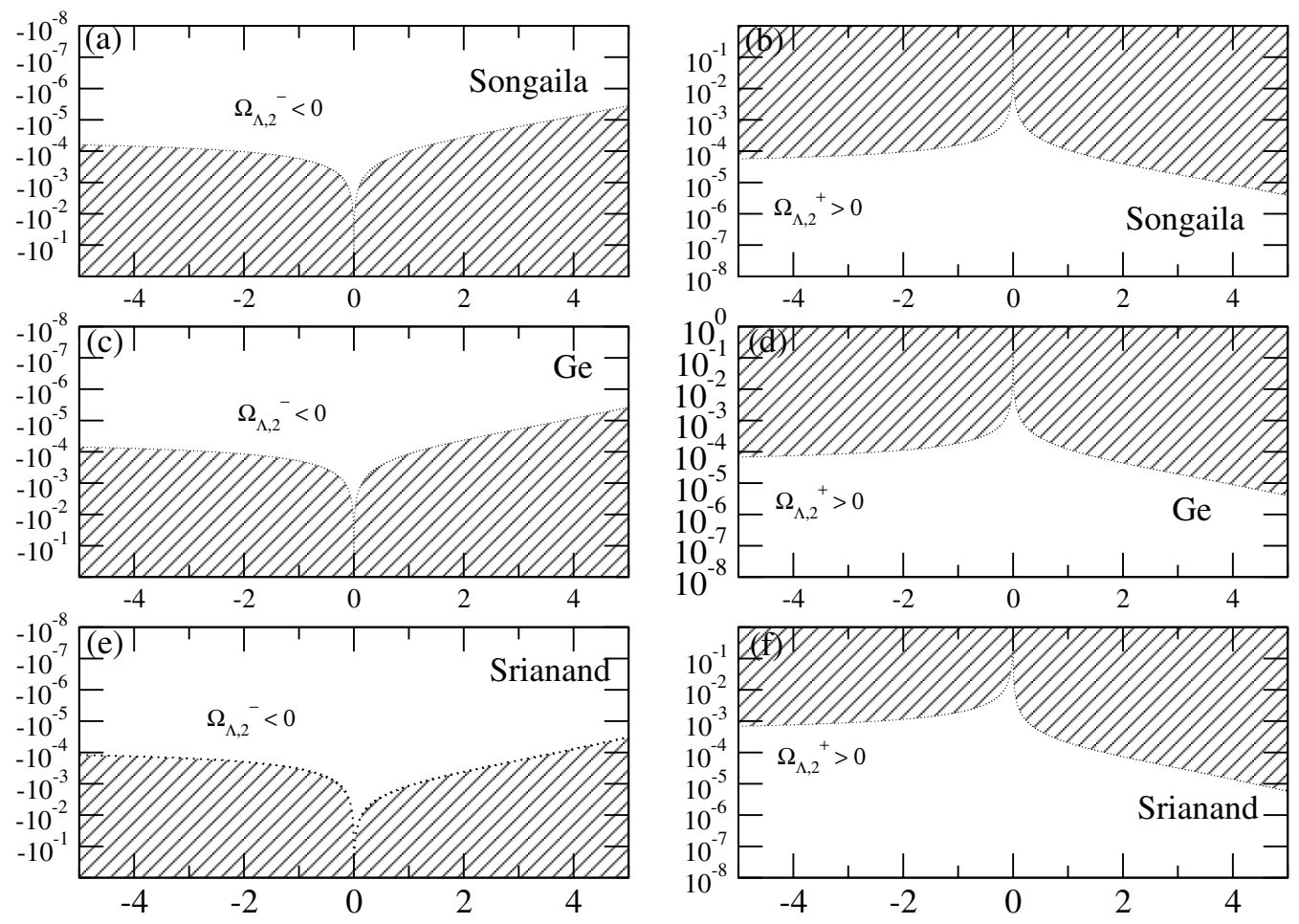

Fig. 3. Bounds on $\Omega_{\Lambda, 2}$ given by the observations. a) and b) corresponds to Songaila observations, c) and d) to Ge observations, e) and f) to Srianand observations. The left panels give the limit on $\Omega_{\Lambda, 2}^{-}$when the right panels are relative to $\Omega_{\Lambda, 2}^{+}$. Cross-hatching indicates the non-allowed regions. The vertical axis corresponds to $\Omega_{\Lambda, 2}$ and the horizontal axis to $m$.

The matter temperature depends on the fraction of electrons ${ }^{4}$. We use the reaction network developped by Galli \& Palla (1998) and couple this primordial kinetic with the hydrodynamic equations (see Puy \& Signore 2002) .

The evolution of the numerical density of baryons is given by

$\frac{\mathrm{d} n_{\mathrm{b}}}{\mathrm{d} t}=-3 H n_{\mathrm{b}}+\left(\frac{\mathrm{d} n_{\mathrm{b}}}{\mathrm{d} t}\right)_{\text {rea }}$,

where $\left(\frac{\mathrm{d} n_{\mathrm{b}}}{\mathrm{d} t}\right)_{\text {rea }}$ describes the contribution of the reactions (see Pfenniger \& Puy 2003). All of these equations depend on the Hubble parameter defined by:

$$
\begin{aligned}
H^{2}= & H_{0}^{2}\left[\left(\Omega_{\mathrm{r}, 0}+\Omega_{\mathrm{r}, \Lambda}\right)(1+z)^{4}+\Omega_{\mathrm{m}, 0}(1+z)^{3}\right. \\
& \left.+\Omega_{\Lambda, 1}+\Omega_{\Lambda, 2}(1+z)^{m}\right]
\end{aligned}
$$

where the redshift is expressed by

$$
\frac{\mathrm{d} z}{\mathrm{~d} t}=-H(1+z)
$$

The numerical integration is a typical initial value problem for stiff differential equations (see Hindmarsh \& Petzold 1995). We start our integration at redshift $z_{\mathrm{i}}=10^{4}$ which corresponds to the age of the Universe $t_{\mathrm{i}}$ :

$t_{\mathrm{i}}=\int_{z_{\mathrm{i}}}^{\infty} \frac{\mathrm{d} z}{H(z)(1+z)}$.

\footnotetext{
${ }^{4}$ Which depends on the reactions of recombination and photoionization coupled with the reactions of charge exchange. All of these reaction rates depend on the temperature and density (see Pfenniger \& Puy 2003).
}

Table 2. Model parameters of runs and initials temperature and time; $T_{\mathrm{r}}$ is in $\mathrm{K}$ and $t_{\mathrm{i}}$ is in years.

\begin{tabular}{ccccc}
\hline \hline Run & $\Omega_{\Lambda, 2}$ & $m$ & $T_{\mathrm{r}}$ at $z_{\mathrm{i}}=10^{4}$ & $t_{\mathrm{i}}$ \\
\hline RUN-1 & $10^{-6}$ & $3 / 2$ & 27203 & 6913 \\
RUN-2 & $-10^{-5}$ & $3 / 2$ & 27736 & 6691 \\
RUN-3 & $10^{-4}$ & -1 & 28775 & 6278 \\
RUN-4 & $10^{-5}$ & -2 & 27524 & 6754 \\
RUN-5 & $5 \times 10^{-5}$ & -4 & 29120 & 6152 \\
RUN-6 & $10^{-5}$ & 2 & 26385 & 7261 \\
RUN-7 & $-5 \times 10^{-5}$ & 2 & 30684 & 5613 \\
RUN-A & $10^{-4}$ & $3 / 2$ & 19674 & 10940 \\
\hline
\end{tabular}

We have developed seven runs (see Table 2) plus one run (RUN-A) illustrating $\left(m, \Omega_{\Lambda, 2}\right)$ parameters of non-allowed regions (see Fig. 5 and condition 42). For each run, through a careful spline approach, we have compared the temperatures with the ones of the standard case (i.e. $\Omega_{\Lambda}=$ constant).

Figure 6 shows the ratio of temperatures between a $\Lambda$-run and the standard case (i.e. $m=0$ ). The difference is negligible for RUN-1, RUN-2 and RUN-3 and a few $10 \%$ for the rest (except the large difference for RUN-A).

These differences can affect the recombination mechanisms and the thermal decoupling between matter and radiation. We define the redshift of recombination $z_{\mathrm{rec}, \mathrm{X}^{\mathrm{n}}}$, the epoch where the abundances $n_{\mathrm{X}^{n+}}=n_{\mathrm{X}^{(n+1)+}}$. We introduce the decoupling redshift $z_{\text {dec }}$ below which the ratio $T_{\mathrm{m}} / T_{\mathrm{r}}$ is lower than 0.99 . 

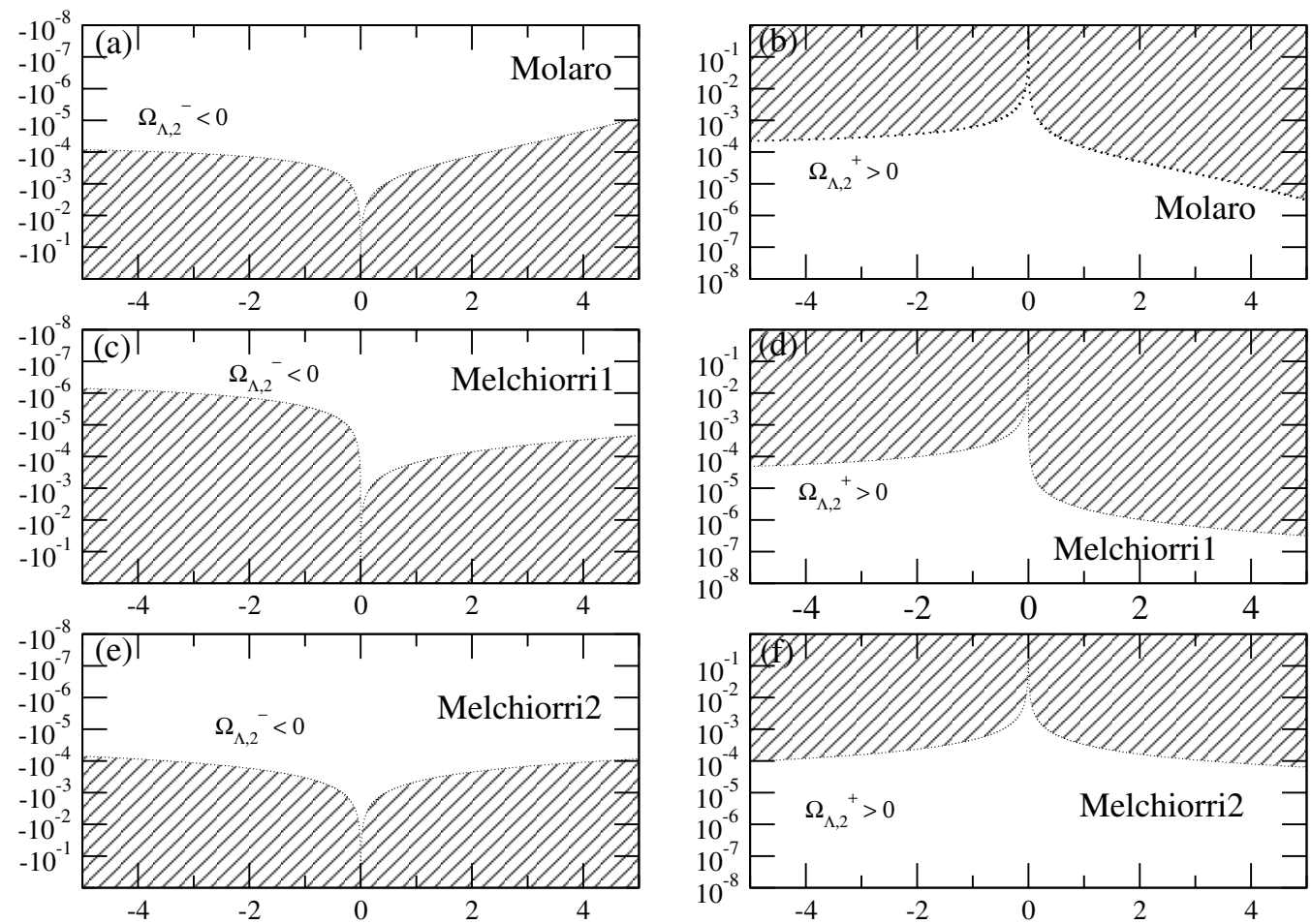

Fig. 4. Bounds on $\Omega_{\Lambda, 2}$ given by the observations. a) and b) correspond to Molaro observations, c) and d) to Melchiorri1 observations, e) and f) to Melchiorri2 observations. The left panels give the limit on $\Omega_{\Lambda, 2}^{-}$, the right panels are relative to $\Omega_{\Lambda, 2}^{+}$. Cross-hatching indicates non-allowed regions. The vertical axis corresponds to $\Omega_{\Lambda, 2}$ and the horizontal axis to $m$.
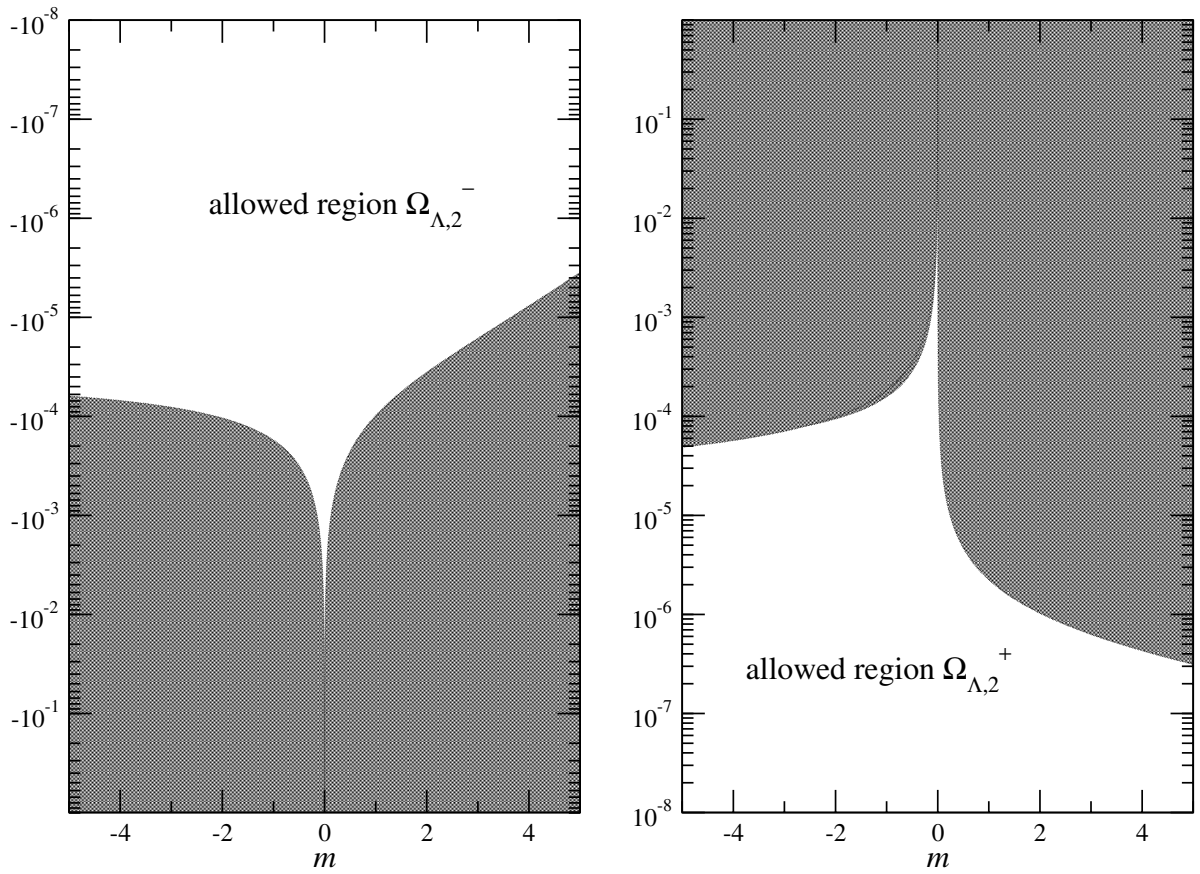

Fig. 5. Total bounds on $\Omega_{\Lambda, 2}$ given by the observations. The left panel gives the limit on $\Omega_{\Lambda, 2}^{-}$and the right panels is relative to $\Omega_{\Lambda, 2}^{+}$. The black area represents the excluded regions. The vertical axis corresponds to $\Omega_{\Lambda, 2}$ and the horizontal axis to $m$.

Table 3 summarizes the different redshifts of recombination and decoupling. $\mathrm{He}^{2+}$ recombination is in the range of redshift $z=5365$ and $z=6320$ with the extreme case $z=8695$ (RUN-A), $\mathrm{He}^{+}$recombination is between $z=2291$ and $z=2696\left(z=3701\right.$ for the RUN-A), $\mathrm{D}^{+}$and $\mathrm{H}^{+}$recombination is between $z=1255$ and $z=1477(z=2024$ for the
RUN-A). We find that the redshift of decoupling is in the range of redshift $z=633$ and $z=811$ ( $z=1258$ for the RUN-A).

Changes in recombination and decoupling could affect the formation of the primordial molecules in the postrecombination Universe. Recently Hashimoto et al. (2003) investigated the primordial chemistry and formation of the first 

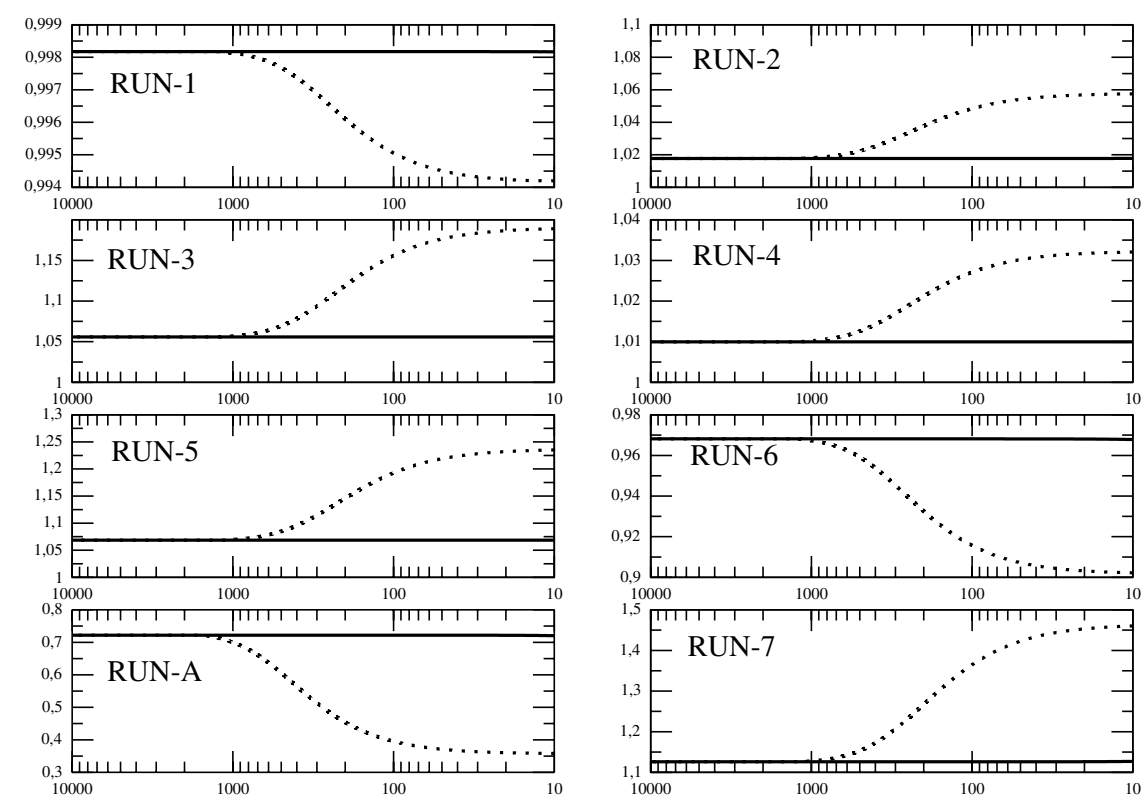

Fig. 6. Ratio of temperatures between a $\Lambda$-run and the standard case $\Omega_{\Lambda}=$ constant. Bold curves represent the ratio of radiation temperatures, dotted curves is the ratio of matter temperatures.

Table 3. Redshifts of recombination and thermal decoupling for the different runs.

\begin{tabular}{cccccc}
\hline \hline Run & $z_{\text {rec, } \mathrm{He}^{2+}}$ & $z_{\text {rec, } \mathrm{He}^{+}}$ & $z_{\text {rec, } \mathrm{D}^{+}}$ & $z_{\text {rec, } \mathrm{H}^{+}}$ & $z_{\mathrm{dec}}$ \\
\hline RUN-1 & 6114 & 2608 & 1429 & 1426 & 773 \\
RUN-2 & 5984 & 2554 & 1399 & 1399 & 749 \\
RUN-3 & 5752 & 2456 & 1345 & 1344 & 704 \\
RUN-4 & 6036 & 2576 & 1410 & 1410 & 755 \\
RUN-5 & 5678 & 2424 & 1328 & 1327 & 690 \\
RUN-6 & 6320 & 2696 & 1477 & 1476 & 811 \\
RUN-7 & 5365 & 2291 & 1255 & 1255 & 633 \\
RUN-A & 8695 & 3701 & 2024 & 2024 & 1258 \\
\hline
\end{tabular}

objects in a model with a decaying cosmological term. They found that molecular formation is significantly shifted to an earlier epoch by $\Delta z \sim 1000$ compared to the standard case. Nevertheless, in our analysis, the decaying cosmological term $\Omega_{\Lambda}=0.7+1.5 \times 10^{-4} a^{-1.5}$, considered by Hashimoto et al. (2003), is ruled out, see Fig. 5.

\section{Conclusion}

In this work, we have quantitatively shown that the allowed values of $\Omega_{\Lambda, 2}$ and $m$ are very low. Thus to give a thermal interpretation of the cosmological constant through a decaying vacuum scenario seems to have stringent limits as determined by the recent analysis of the CMBR temperature at different redshifts.

Measurements from the analysis of the carbon finestructure population ratio are particularly difficult because of numerous sources of excitation. For example, fluorescence and dust emission are possible in the population of the excited levels. As Songaila et al. (1994) mentioned, it would be gratifying to find an appropriate $\mathrm{C}$ absorber at higher redshift to improve existing, much less sensitive, limits from $\mathrm{C}^{+}$.

The multi-frequency measurements through the SZ effect are the most promising. Precise values of $T_{\mathrm{CMBR}}$, with a significantly larger number of targets (i.e. clusters), could be obtained by this method. In this context several projects are planned, such as the upgraded MITO (see Lamagna et al. 2002) or the next balloon-borne telescope to measure the cosmic microwave radiation (or OLIMPO experiment, see Masi et al. 2003), and could lead to interesting constraints on $\Omega_{\Lambda, 2}$.

Acknowledgements. This work was supported by the Swiss National Science Foundation and the University of Geneva. The author acknowledges Daniel Pfenniger, Monique Signore, Norbert Straumann and Patrick Vonlanthen for fruitful discussions on $\Lambda$-cosmologies, Francesco Melchiorri and Paolo Molaro for communications on CMBR temperature measurements. Special thanks to Agnese Blasina-Puy.

\section{References}

Bahcall, J., \& Wolf, R. 1968, ApJ, 152, 701

Barger, V., Kneller, J., Lee, H., Marfatia, D., \& Steigmann, G. 2003, Phys. Lett. B, 566, 8

Battistelli, E., de Petris, M., Lamagna, L., et al. 2003, Mem. S. A. It. 74,316

Bennett, C., Halpern, M., Hinshaw, G., et al. 2003, ApJS Lett., 148, 1

Bloomfield Torres, L., \& Waga, I. 1996, MNRAS, 279, 712

Caldwell, R., Dave, R., \& Steinhardt, P. 1998, Phys. Rev. Lett., 80, 1582

Chen, W., \& Wu, Y. 1990. Phys. Rev. D, 41, 695

de Bernardis, P., Ade, P. A. R., Bock, J. J., et al. 2000, Nature, 404, 955

Danese, L., \& de Zotti, G. 1977, Riv. Nuovo Cimento, 7, 277

Dvali, G., Gabadze, G., \& Porrati, M. 2000, Phys. Lett. B, 485

Einstein, A. 1917, Sitzungber. Preuss. Akad. Wiss. Phys. Math. Kl., 142,177 
Elizalde, E., Lidsey, J., Nojiri, S., \& Odintsov, S. 2003, Phys. Lett. B, 574,1

Fabbri, R., Melchiorri, F., \& Natale, V. 1978, Ap\&SS, 59, 223

Freedman, W. 2000, Phys. Rep., 333, 13

Freese, K., Adams, F. C., Frieman, J. A., Mottola, E., et al. 1987, Nucl. Phys. B, 287, 797

Freese, K., \& Lewis, M. 2002, Phys. Lett. B, 540, 1

Frolov, A., Kofman, L., \& Starobinsky, A. 2002, Phys. Lett. B, 545, 8

Galli, D., \& Palla, F. 1998, A\&A, 335, 403

Gasperini, M. 1987, Phys. Lett. B, 194, 347

Ge, J., Bechtold, J., \& Black, J. 1997, ApJ, 474, 67

Hashimoto, M., Kamikawa, T., \& Arai, K. 2003, ApJ, 598, 13

Hindmarsh, A., \& Petzold, L. 1995, Comput. Phys., 9, 34

Inagaki, T., Meng, X., \& Murata, T. 2003 [arXiv:hep-th/0306010]

Kamenshchik, A., Moschella, U., \& Pasquier, V. 2001, Phys. Lett. B, 511,265

Kazanas, D. 1980, ApJ, 241, L59

Kimura, K., Hashimoto, K., Sakoda, K., \& Arai, K. 2001, ApJ, 561, L19

Kompaneets, A. 1957, Sov. Phys. JETP, 4, 430

Lamagna, L., de Petris, M., Melchiorri, F., et al. 2002, in Experimental Cosmology at Millimetre Wavelengths, ed. M. de Petris, \& M. Gervasi, AIP Conf. Proc., 616, 92

Levshakov, S., Takahara, F., \& Agafonova, I. 1999 ApJ, 517, 609

Mangano, G., Miele, G., Pastor, S., \& Peloso, M. 2002, Phys. Lett. B, 534,8
Masi, S., Ade, P., de Bernardis, P., et al. 2003, Mem. S. A. It., 74, 96 Mather, J., et al. 1999, ApJ, 512, 51

Matyjasek, J. 1995, Phys. Rev. D, 51, 4154

Molaro, P., Levshakov, S. A., Dessauges-Zavadsky, M., \& D’Odorico, S. 2002, A\&A, 381, L64

Meng, X., \& Wang, P. 2003 [arXiv:astro-ph/0308284]

Overduin, J., Wesson, P., \& Bowyer, S. 1993, ApJ, 404, 1

Overduin, J., \& Cooperstock, F. 1998, Phys. Rev. D, 58, 043506

Özer, M., \& Taha, M. 1986, Phys. Lett. B, 171, 363

Özer, M. 1999, ApJ, 520, 45

Peebles, P. J. E. 1968, ApJ, 153, 1

Peebles, P. J. E., \& Ratra, B. 2003, Rev. Mod. Phys., 75, 559

Perlmutter, S., Aldering, G., Goldhaber, G., et al. 1999, ApJ, 517, 565

Pfenniger, D., \& Puy, D. 2003, A\&A, 398, 447

Puy, D., Alecian, G., Le Bourlot, J., Leorat, J., \& Pineau Des Forets, G. 1993, A\&A, 267, 337

Puy, D., \& Signore, M. 2002, New Ast. Rev., 46, 709

Ratra, B., \& Peebles, P. J. E. 1988, Phys. Rev. D, 37, 3406

Riess, A., Filippenko, A. V., Challis, P., et al. 1998, AJ, 116, 1009

Sahni, V., \& Starobinsky, A. 2000. Int. J. Mod. Phys. D, 9, 373

Silveira, V., \& Waga, I. 1994, Phys. Rev. D, 50, 4890

Songaila, A., Cowie, L. L., Vogt, S., et al. 1994, Nature, 371, 43

Srianand, R., Petitjean, P., \& Ledoux, C. 2000, Nature, 408, 931

Straumann, N. 1999, Eur. J. Phys., 419, 427

Waga, I. 1993, ApJ, 414, 436

Weinberg, S. 1989, Rev. Mod. Phys., 61, 1 